\title{
Analysis of the Detection Effects in Coal Mine Goaf by Using Large Fixed Source Loop TEM Method
}

\author{
Tao Liu ${ }^{1, a^{*}}$, Jiao Luo ${ }^{1, b}$ and Long Zhang ${ }^{1, c}$ \\ ${ }^{1}$ College of Geology Engineering and Geomatics, Chang'an University, Xi'an, China

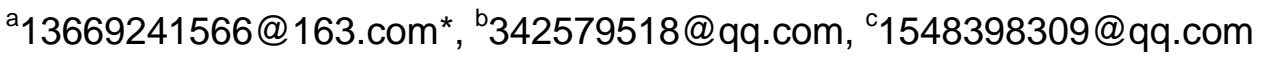

Keywords: TEM; Large Fixed Source Loop; Goaf; Apparent Resistivity Profile.

\begin{abstract}
In this paper, under the geophysical prerequisite of low resistivity anomaly zone being the main characteristic of the goaf, researchers have used large fixed source loop transient electromagnetic method (referred to as TEM) to prospect the goaf in a coal mine of Shanxi Province. After a series of processing, the author writes a MATLAB program to calculate the all-time apparent resistivity, so researchers can map the all-time apparent resistivity profile and make the integrated interpretation or analysis. It is speculated that the south, northwest and northeast of the exploration zone have large goaf areas, and a smaller goaf is distributed in the center of the exploration zone. Party A should pay enough attention to these goaf areas and should make drilling verifications in time.
\end{abstract}

\section{Introduction}

After the coal mining, the coal mine goaf often causes great harms to the normal production of coal mine. In the exploration of coal mine goaf, different strata have different lithology and electrical characteristics. In the goaf area, the rock structure surrounded by the goaf has changed, so its apparent resistivity will have certain differences with the surrounding rocks. The goaf cavity is always against the current, showing the relatively high resistances on the ground detections $[1,2]$. However, when the goaf is collapsed and filled with water, it will show a relatively low resistance. Before the coal mine goaf is collapsed, it is often difficult to predict. On the basis of electrical difference, the large loop source Transient Electromagnetic Method detection technology has many advantages, such as great exploration depth, high effects, penetration into the high resistance shielding layer and high lateral resolution, etc. It is sensitive to coal mine goaf and has played good effects in the detection on the coal mine goaf $[3,4]$.

\section{The Principles of TEM}

Transient electromagnetic method (referred to as TEM) is a kind of electromagnetic detection method in time domain. It uses no grounding loop or electric dipole source to send the primary field underground and observe the secondary vortex field during the break of the primary field $[5,6]$. The basic working method is to lay a transmitter coil with certain current waveform, so as to produce magnetic field in the surrounding space, and produce the induced current in conductive rock underground. Due to the heat loss, when the power is off, the induced current declines over time and this declining process is divided into early and late stages. Early frequency electromagnetic field is 
equal to the high component in frequency domain, with the declining fast and small skin depth $[7,8]$. On the contrary, the late component is equal to the low frequency components in frequency domain, with slow declining and big skin depth. By measuring the secondary field variation law with time, when the power is off, researchers can get different geoelectrical characteristics. This method has many advantages, for instance, small volume effect, high rate of transverse and sensitivity to low resistance response, etc. According to the characters of the declined curve for secondary field, researchers can judge the electrical, scale and occurrence to the geological body underground $[9,10]$.

\section{TEM Data Collection in the Wild}

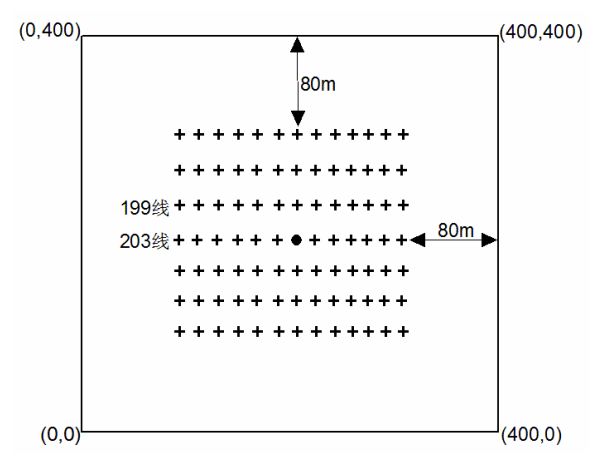

Fig. 1 Schematic Diagram of Loop an Measuring Point Location

The schematic diagram of loop and measuring point location are shown in Fig. 1. Through field experiments, researchers can determine the following parameters: loop frame size is $400 \mathrm{~m} * 400 \mathrm{~m}$; emission frequency is $25 \mathrm{~Hz}$; superposition times is 256 ; sampling window time is 0.036 to 27.915 milliseconds; measuring channel number is 40 ; the emission current is about $3.5 \mathrm{~A}$; equivalent area of the receiving coil is $2200 \mathrm{~m}^{2}$. The quality inspection results meet the relevant specification and design requirements, and the data collection is reliable and has high quality.

\section{Data Processing}

For the original measured data, the data sorting and pretreatment (data smooth, eliminate bad way) is the first step, then a MATLAB program should be written to calculate the all-time apparent resistivity. After a series of processing, the details are as follows, the time-depth transformation, the terrain correction, filtering and bleaching. With the final data, researchers can map the all-time apparent resistivity profile and make the integrated interpretation or analysis. The processing picture is shown in Fig. 2. 


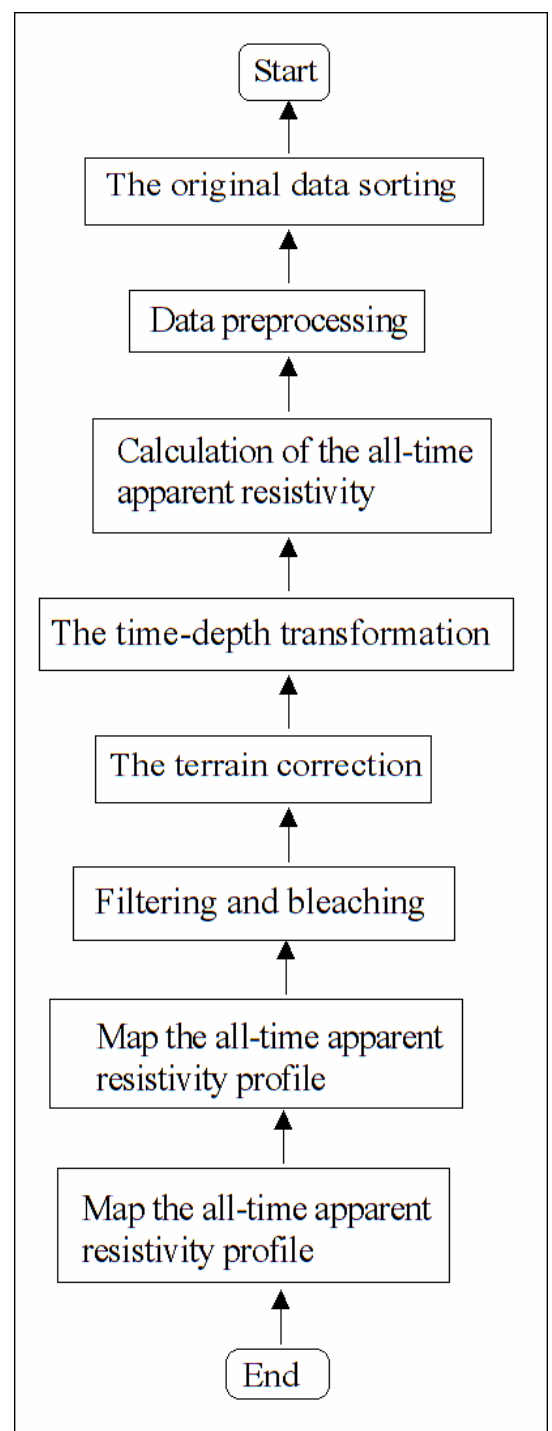

Fig. 2 Schematic Diagram of Loop and Measuring Point Location

\section{TEM Apparent Resistivity Profile Interpretation and Analysis}

There are many measuring lines in the work area, so it is difficult to put profiles to all lines in the report. Therefore, researchers selected some typical profiles to make relevant interpretation, explaining the abnormal regularity of the goaf. 


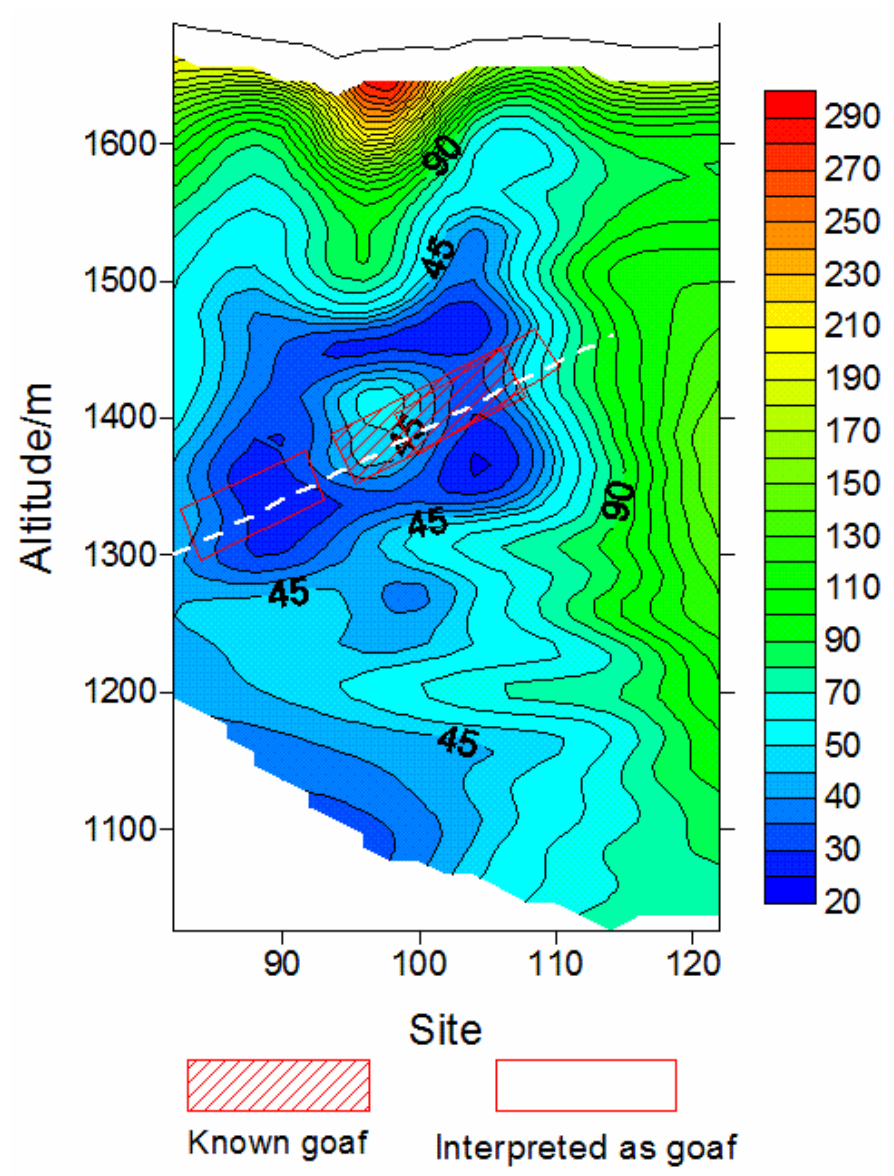

Fig. 3 The All-time Apparent Resistivity Profile for Line 13

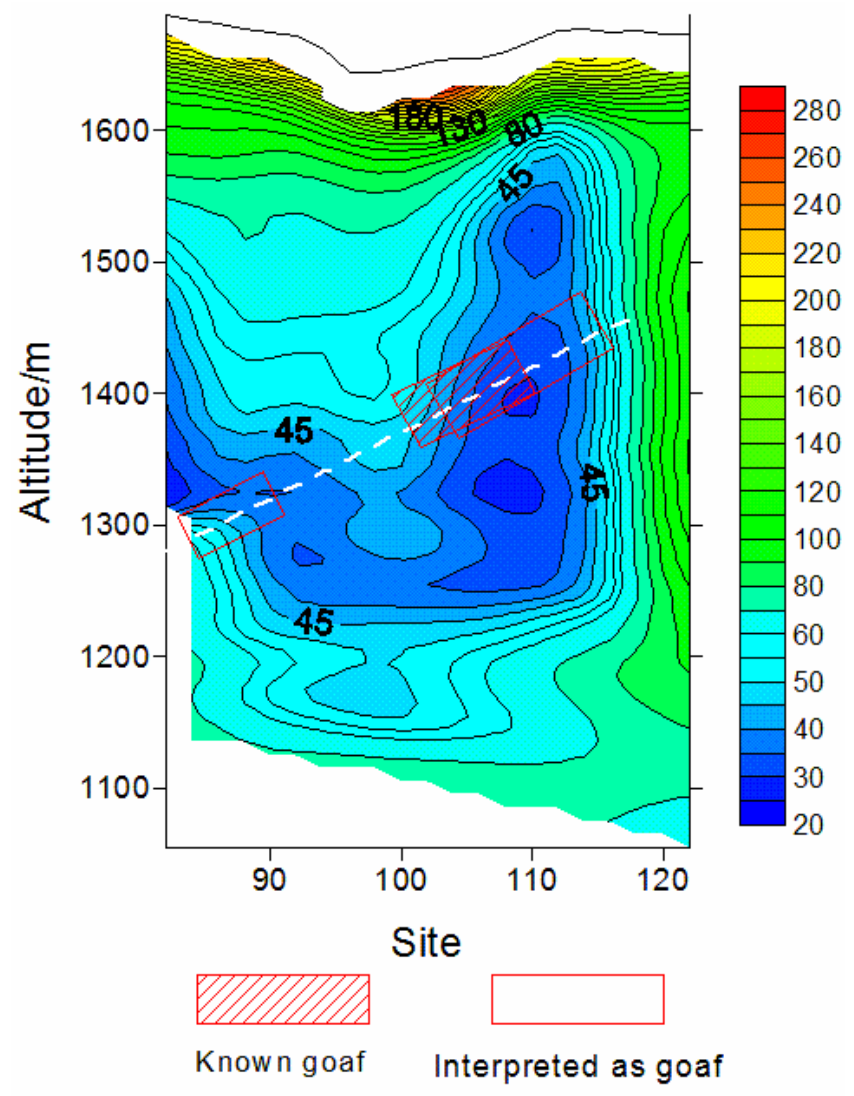

Fig. 4 The All-time Apparent Resistivity Profile for Line 14 
Fig. 3 and Fig. 4 are the all-time apparent resistivity profile for line 13 and line 14, in which the white dotted line is the contour for coal floor. In order to better analyze the profile abnormality of TEM and relevance between lines, researchers selected the adjacent line 13 and line 14 to make interpretation and analysis. Horizontally, the apparent resistivity contours of the two lines are layered distribution overall, which conform to the stratigraphic structure. The local contour is bending upwards. For example, points 82 to 92 and points 96 to 108 period of the line 13, points 82 to 88 and points 102 to 118 period of the line 14. Among them, points 96 to 108 period of the line 13 and points 102 to 118 period of the line 14 correspond to the known goaf very well, with points 82 to 92 periods of the line 13 and points 82 to 88 period of the line 14 being interpreted as goaf. From shallow to deep, the apparent resistivity is the high-middle-low-middle mode in the electrical characteristics. The surface is mainly the quaternary loess, so the resistivity is higher. With downward extension, the resistivity decreases gradually and coal seam is characterized by middle-high resistance. Downward the Yanchang group of Jurassic formation, resistivity is of middle-low resistance. Overall, the correlation of the two lines is better and the anomalies are more reliable.

\section{Summary}

Under the geophysical prerequisite of low resistivity anomaly zone being the main characteristic of the goaf, researchers used large fixed source loop transient electromagnetic method to prospect the goaf in a coal mine of Shanxi Province. For line 13 and line 14, the apparent resistivity profile processing results show that the actual geoelectric section corresponds to the profile very well, so researchers can do further detailed interpretation. On one side, it is speculated that there are large goaf areas in the south, northwest and northeast of the exploration zone. On the other side, there is a smaller goaf area in the center of the exploration zone. Party A should pay enough attention to these areas, which are interpreted as goaf and make drilling verification in time. It is worth noting that according to the principle, low resistivity anomaly zone is the main characteristic, and researchers can select the scope of goaf area. Besides, the influence factors of the resistivity are numerous. In addition to the complexity of the geological factors and uncertainty of geophysical interpretation, the results speculated by the researchers may be deviated from the actual situation, so it also needs party A to drill or validate other geophysical exploration methods.

\section{References}

[1]Zhou. ,S., Geophysical exploration method in gob application effect: Science and Technology in Western China, , 2008, pp50-51.

[2]Zhao, L., Y. Zhao, and D. Huang, Coal seam collapse column in high-resolution seismic exploration technology, Coal Geology of China, 2006, pp 52-53.

[3]Liu Xiaobo, Cui Jingbin, Liu Jianhong, Identifying Coal Mine Collapse Columns and Gobs Utilizing 3D Seismic Interpretation, Society of Exploration Geophysicists, 2012,pp4609-4614. 
[4] Spies B R., A Field Occurance of Sign Reversals with the Transient EM Method, Geophy Prosp, 1980, pp620-632.

[5] Lee T J., Transient Electromagnetic Response of a Polarizable Ground, Geophysics, 1981, pp1037-1041.

[6] Guo-Qiang, X., and L. Xiu, The Technology of TEM Tunnel Prediction Imaging: Chinese Journal of Geophysics, 2008, no. 03, pp 894-900 (in Chinese).

[7] Hua-rong, P., Electromagnetic Sounding Theory: Geological Publishing House, 1990,pp 1-10.

[8] Li, X., W. Junjie and C. Daming, Advanced Geologic Forecasting for Unfavorable Geological Body with Water-Transient Electromagnetic Method, Journal of Geotechnical Investigation \&Surveying, 3, 70275.

[9] Shu-cai, L., L. Shu-chen, and Z. Qing-song, Forecast of Karst-fractured Groundwater and Defective Geological Conditions, Chinese Journal of Rock Mechanics and Engineering, 2007, no. 2, pp217-225.

[10] Shu-cai, L. I., X. Yi-guo, and Z. Qing-song, Key Technology Study on Comprehensive Prediction and Early-warning of Geological Hazards during Tunnel Construction in High-risk Karst Areas, Chinese Journal of Rock Mechanics and Engineering, 2008, pp1297-1307. 